\title{
Just the Facts: Point-of-care ultrasound in the management of shoulder dislocations
}

\author{
Ryan Henneberry, MD, CCFP(EM), FCFP*; Tara Dahn, MD, MASc, CCFP(EM)*; \\ Paul Atkinson ${ }^{\circ}, \mathrm{MB}$ BCh BAO, $\mathrm{MA}^{\dagger}$ \\ CLINICAL SCENARIO
}

A 59-year-old man presents with left shoulder pain after falling while playing with his dog at the park. He drove himself to the emergency department (ED). He reports $5 / 10$ pain and has reduced range of motion of the shoulder. His shoulder looks normal on exam and is not squared off. You wonder if he might have a posterior shoulder dislocation.

\section{KEY CLINICAL QUESTIONS}

1. How accurate is the clinical examination and plain radiography for diagnosing posterior shoulder dislocation?

Together, clinical examination and radiographs can miss a significant proportion of posterior shoulder dislocations. Posterior shoulder dislocations are much less common than anterior shoulder dislocations, accounting for $2-5 \%$ of shoulder dislocations seen in the ED. ${ }^{1}$ Posterior shoulder dislocations are also much more difficult to diagnose clinically and radiographically. It has been reported that posterior shoulder dislocations were missed on initial clinical exam in $79 \%$ of cases. ${ }^{2}$ Studies have also shown that up to $50-88 \%$ of posterior shoulder dislocations can be missed on initial anterior-posterior (AP) radiographs. ${ }^{3}$ Missing a posterior shoulder dislocation can result in delayed treatment and can increase the risk of the patient requiring a surgical reduction.

Several radiographic signs have been described to increase the suspicion for posterior shoulder dislocation, but none are present in all cases and many are not specific. A recent study found that a reverse Hill-Sachs lesion, fixed internal rotation of the humeral head, and widened joint line were present in 83,76 , and $37 \%$ of posterior shoulder dislocations, respectively. ${ }^{1}$ Adding an additional orthogonal view is critical to improve the sensitivity of radiographs for posterior shoulder dislocation, with the preferred view being an axillary view. Unfortunately, an axillary view can be difficult to obtain in patients with posterior shoulder dislocations due to pain and difficultly abducting the arm. An oblique or scapular "Y" view can be used as an alternative to an axillary view but has been reported to still miss posterior dislocations in a significant number of patients. ${ }^{3}$

2. When clinical examination and radiography are equivocal what other diagnostic tools are available when suspecting shoulder dislocation?

Traditionally, the use of computed tomography (CT) was indicated; however, there is increasing evidence supporting the use of point-of-care ultrasound (POCUS). Classically, CT scan has been used when the diagnosis of shoulder dislocation is in question; however, CT scans are relatively time and resource intensive and also expose the patient to radiation. In settings where access to CT is not timely, this can put the patient with a suspected shoulder dislocation at

From the * Department of Emergency Medicine, Dalhousie University, QEll, Halifax, NS; and the ${ }^{\dagger}$ Department of Emergency Medicine, Dalhousie University, Saint John Regional Hospital, Saint John, NB.

Correspondence to: Dr. Tara Dahn, 1796 Summer Street, Suite 355, Halifax Infirmary, Halifax, NS, B3H 3A7; Email: tara.dahn@dal.ca

(c) Canadian Association of Emergency Physicians 2020

CJEM 2020;22(3):287-290

DOI 10.1017/cem.2020.14 
risk for developing chronic degenerative changes or avascular necrosis of the humeral head, and reduces the chance of successful primary reduction.

POCUS is emerging as a useful tool in musculoskeletal complaints and can be particularly helpful in the diagnosis of posterior shoulder dislocations. ${ }^{4}$

\section{How do you perform POCUS for shoulder dislocation?}

Posteriorly and in a transverse orientation, looking forward at the shoulder joint. A curvilinear (abdominal) probe, or linear probe in children, is placed on the posterior aspect of the shoulder in a horizontal (transverse) orientation (see Figure 1). In a normal shoulder, the humeral head lines up with the glenoid at a similar depth (see Figure 2). An anterior dislocation will result in the humeral head being displaced into the far field (deeper; anteriorly), whereas a posterior dislocation will result in the humeral head being displaced into the near field (more superficial; posteriorly).

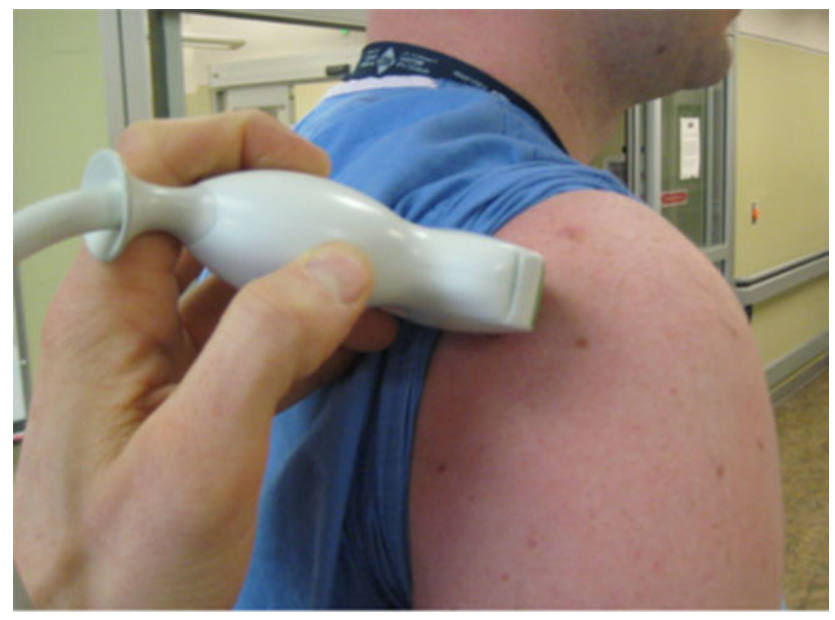

Figure 1. Orientation of ultrasound probe for posterior shoulder imaging.
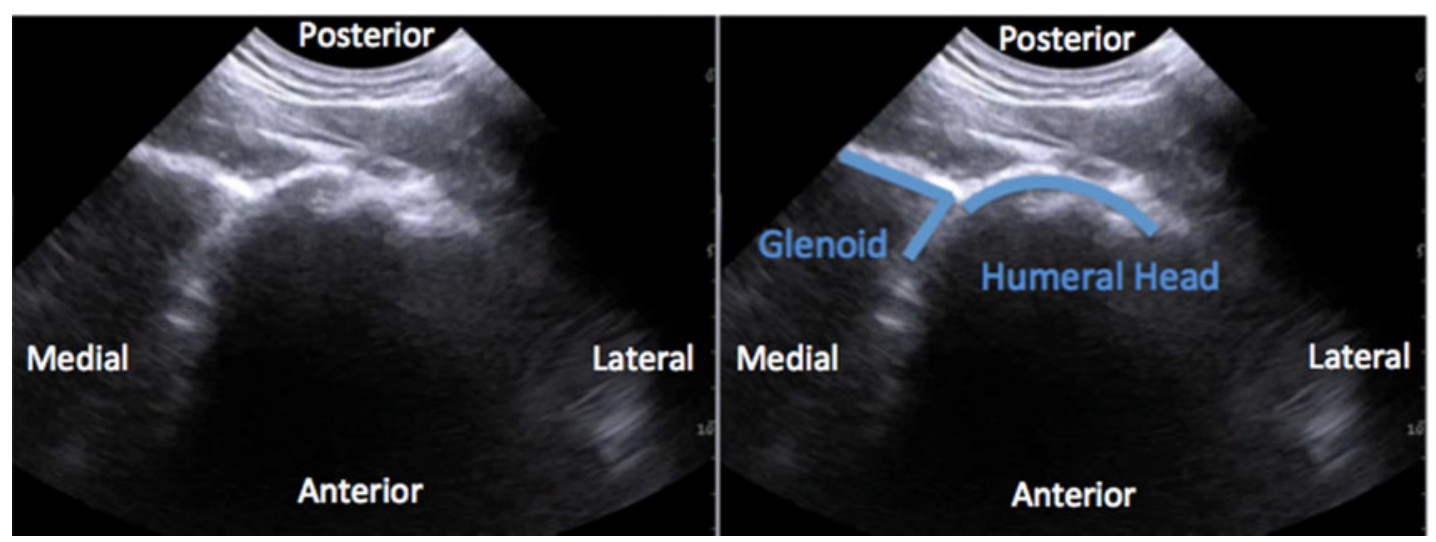

Figure 2. Normal (right) shoulder ultrasound scan (a) and labeled scan showing glenoid lining up at a similar depth with humeral head (b).

Studies have shown that the posterior approach for shoulder POCUS can be learned easily and that even novice ultrasonographers can achieve competence with highly sensitive and specific results. ${ }^{5}$

Although it will not be covered in depth in this article, shoulder POCUS can also be used to assess for a joint effusion in a patient being considered for septic arthritis of the shoulder. The posterior approach described above is well suited for joint effusion assessment and can also be used to assist an US guided joint aspiration of the shoulder. 


\section{Is POCUS useful in management of shoulder dislocations other than confirming the diagnosis?}

Yes, POCUS can also confirm successful reduction. POCUS can provide a dynamic evaluation of the glenohumeral joint, which can give immediate information about whether or not a reduction attempt was successful. This can be helpful for both anterior and posterior shoulder dislocations. Repeat radiography may still be needed to document successful reduction, depending on the practice at your institution, but POCUS could help mitigate delays associated with unsuccessful reduction attempts.

\section{CASE RESOLUTION}

Our patient's plain radiographs identified a posterior shoulder dislocation as well as a large reverse Hill-Sachs lesion (Figures $3 \mathrm{a}$ and $3 \mathrm{~b}$ ). POCUS was performed during the reduction attempt to confirm successful reduction due to the patients challenging clinical exam. Figures $3 \mathrm{c}$ and $3 \mathrm{~d}$ demonstrate the pre- and postreduction POCUS findings. Unfortunately, this patient's shoulder was very unstable and re-dislocated shortly after while in the ED. The patient ultimately required an open reduction and internal fixation in addition to a rotator cuff repair.

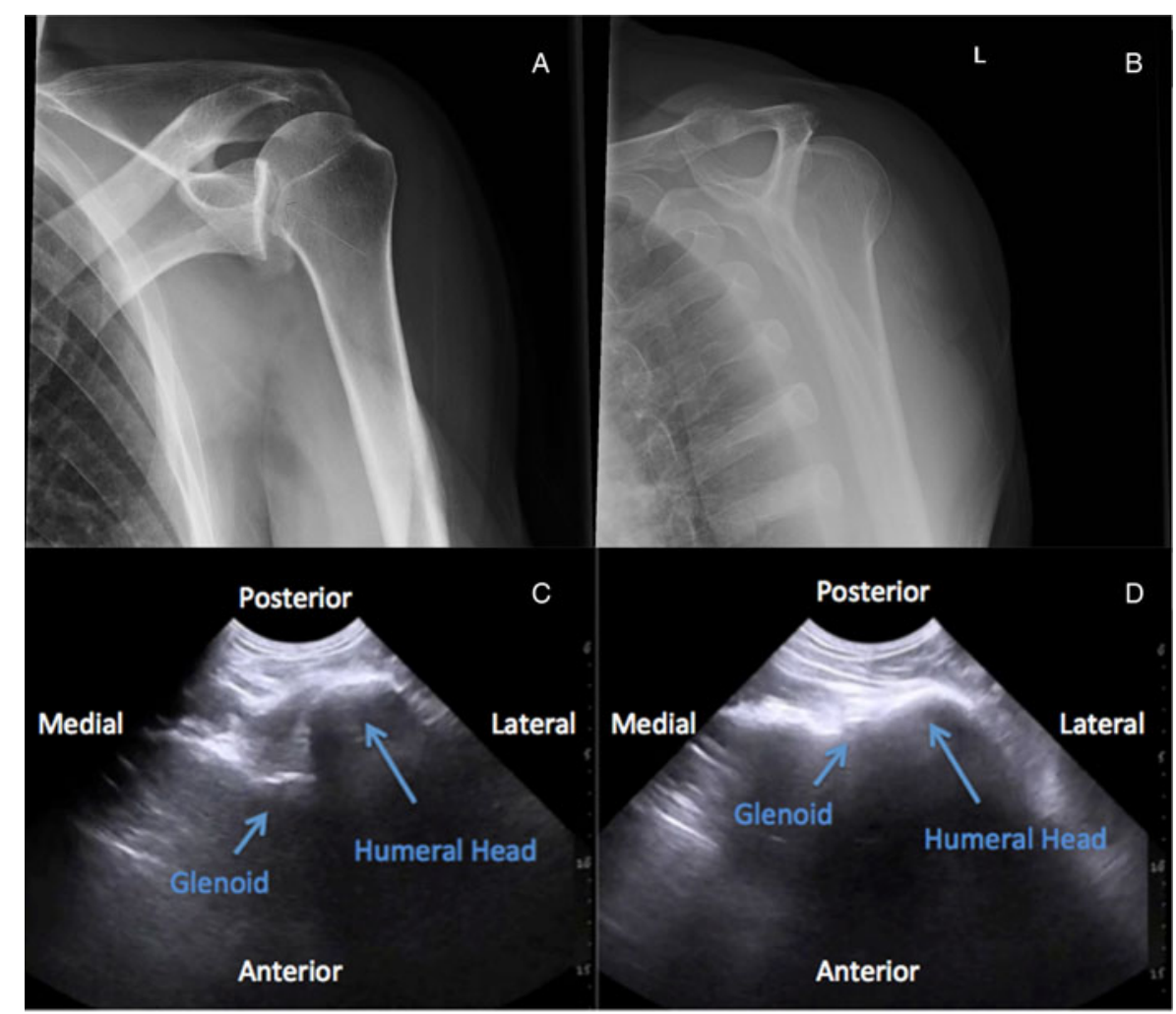

Figure 3. Case Images: (a) Left shoulder AP radiograph showing posterior shoulder dislocation and large reverse Hill-Sachs lesion; (b) scapular "Y" view radiograph showing posterior shoulder dislocation; (c) POCUS showing humeral head located posteriorly relative to the glenoid; and (d) post reduction POCUS showing a successful reduction with humeral head lining up with the glenoid.

Keywords: Emergency medicine, musculoskeletal, ultrasound

Competing interests: Paul Atkinson is lead editor of the textbook Point of Care Ultrasound for Emergency Medicine and Resuscitation (Oxford 2019).

\section{REFERENCES}

1. Gimber LH, Taljanovic MS, Rockov ZA, Krupinksi EA, Chadaz TS, Rogers LF. The veil of obscuration: additional radiographic sign of posterior shoulder dislocation. Acta Med Acad 2018;47(2):165-75. 
Ryan Henneberry et al.

2. Rowe CR, Zarins B. Chronic unreduced dislocations of the shoulder. F Bone foint Surg Am 1992;64(4):494-505.

3. Aparico G, Calyo E, Bonilla L, Espejo L, Box R. Neglected posterior dislocations of the shoulder: controversies on indications for treatment and new CT scan findings. 7 Orthop Sci 2000;5:37-9.

4. Beck S, Chilstrom M. Point-of-care ultrasound diagnosis and treatment of posterior shoulder dislocation. Am 7 Emerg Med 2013;31 (2):449.e3-5.

5. Lahham S, Becker B, Chiem A, et al. Pilot study to determine accuracy of posterior approach ultrasound for shoulder dislocation by novice sonographers. West 7 Emerg Med 2016;17(3):377-82. 